\title{
Generalized Composite Numerical Integration Rule Over a Polygon Using Gaussian Quadrature
}

\author{
M. Alamgir Hossain ${ }^{1}$ and Md. Shafiqul Islam*,2 \\ ${ }^{1}$ Department of Mathematics, Jagannath University, Dhaka -1100, Bangladesh \\ ${ }^{2}$ Department of Mathematics, Dhaka University, Dhaka - 1000, Bangladesh
}

(Received: 22 November 2012; Accepted: 17 July 2013)

\begin{abstract}
The aim of this paper is to evaluate double integrals over a polygon exploiting coordinate transformation. At first any polygon with $m$-sides is decomposed into $(m-2)$ triangles. Then each triangle is transformed into a standard triangular finite element using the basis functions in

local space. Then the standard triangle is decomposed into $4 \times n^{2}$ right isosceles triangles with side lengths $1 / n$, and thus composite numerical integration is employed. In addition, the affine transformation over each decomposed triangle and the use of linearity property of integrals are applied. Finally, each isosceles triangle is transformed into a 2-sqare finite element to compute new $s^{2}$ sampling points and corresponding weight coefficients, using $s$ point's conventional Gaussian quadrature, which are applied again to evaluate the double integral. We demonstrate some numerical examples through the proposed method.
\end{abstract}

Key words: Numerical Integration, Quadrilateral and Triangular Finite Elements, Gaussian Quadrature.

\section{Introduction}

Numerical integrations over triangular regions were first introduced by Hammer et $a l^{1-3}$, and then by Stroud ${ }^{4} *$. In finite element method, the triangular elements are widely used in the area of numerical integration schemes ${ }^{5}$. The works of Hammer et $a l^{1-3}$ have been further developed by Cowper $^{6}$ and thus he provided a table of Gaussian quadrature formulae for symmetrically placed integration points. Lethor ${ }^{7}$ and Hillion ${ }^{8}$ derived formulas for triangles as product of one-dimensional Gauss quadrature rule. Laursen and Gellert $^{9}$ also discussed elaborately symmetric integration formulae of precision up to degree ten. One may realize that a lot of works of numerical integration using Gaussian quadrature over triangular ${ }^{1-9}$ region, composite numerical integration using Gauss quadrature over triangular $^{10-12}$ region and over quadrilateral ${ }^{13}$ region have been done, but no generalized work of composite numerical integration has been attempted so far over the polygon.

Recently, a rigorous and elaborate survey has been reported by Sarada and Nagaraja ${ }^{14}$. In this paper, they have derived some formulas for limited shapes of triangles and quadrilaterals, and then generalized their process for any arbitrary polygon. This work is based on higher order (e.g., 5, 10, 15 and 20) Gaussian quadrature rule. In contrast to this study, we propose to develop a general composite integration formula over any arbitrary polygon by decomposing the polygon into arbitrary shapes of triangles, described in section II, using lower order (e.g., 3, 5 and 10) Gauss points to get more accuracy. Numerical examples are taken from the recent paper ${ }^{14}$, and thus are compared. The subsequent formulations are developed by Mathematica.

\section{Formulation of Integrals Over an Arbitrary Polygon}

The integral of an arbitrary function, $f(x, y)$ over an arbitrary polygon $A P$ with $m$ sides is given by

$$
I=\iint_{A P} f(x, y) d y d x=\iint_{A P} f(x, y) d x d y
$$

At first the polygon $A P$ with $m$-sides is decomposed into $m$ 2 triangles shown in figure 1. The integral $I$ of Eq. (1) is the sum of $m-2$ integrals whose domains are triangles.

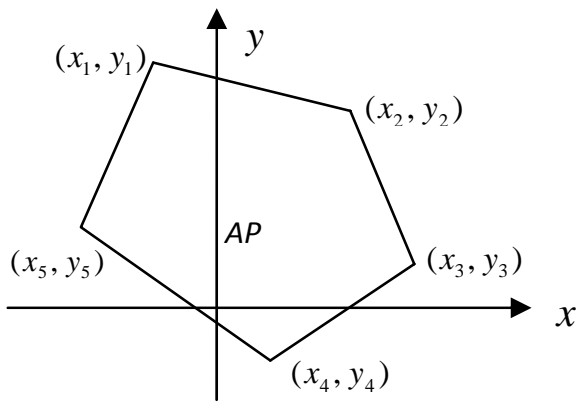

(a) Arbitrary polygon $A P$

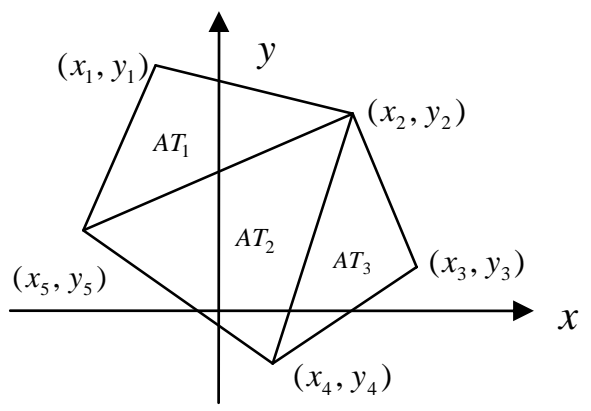

(b) Decomposed triangles $A T_{\mathrm{t}}$

Fig. 1. Polygon $A P$ with $m$-sides is decomposed into $m-2$ triangles

$I=\sum_{t=1}^{m-2} I_{t}=\sum_{t=1}^{m-2} \iint_{A T_{t}} f(x, y) d y d x=\sum_{t=1}^{m-2} \iint_{A T_{t}} f(x, y) d x d y$

The each integral $I_{t}$ of Eq. (2) is then transformed into an integral over the region of the standard triangle $S T=\{(u, v):-1 \leq v \leq 1,-1 \leq u \leq-v\} \quad$ by the liner triangular finite element basis functions $L_{i}(u, v)$, shown in Fig. 2: 


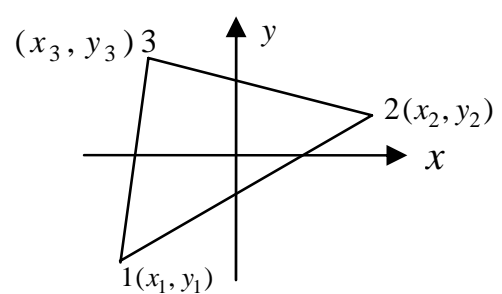

(a) Arbitrary triangle $A T_{\mathrm{t}}$

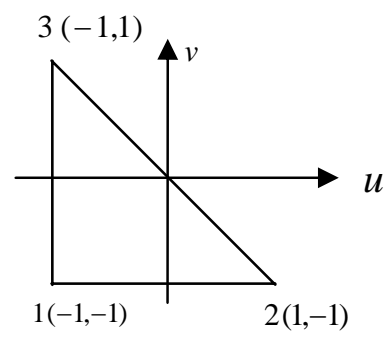

(b) Standard triangle $S T$

Fig. 2. Transformation of arbitrary triangle $A T_{\mathrm{t}}$ into equivalent standard triangle $S T$

$L_{1}(u, v)=-\frac{1}{2}(u+v), L_{2}(u, v)=\frac{1}{2}(1+u)$ and $L_{3}(u, v)=\frac{1}{2}(1+v)$.

The coordinates are changed by assuming that

$$
x=\sum_{i=1}^{3} x_{i} L_{i} \text { and } y=\sum_{i=1}^{3} y_{i} L_{i}
$$

and the corresponding Jacobian

$$
J_{1}=\frac{\partial(x, y)}{\partial(u, v)}=\frac{\partial x}{\partial u} \frac{\partial y}{\partial v}-\frac{\partial x}{\partial v} \frac{\partial y}{\partial u}
$$

Therefore using Eqs. (3) and (2) to obtain,

$$
I_{t}=\iint_{A T_{t}} f(x, y) d y d x=\iint_{S T} f(u, v)\left|J_{1}\right| d u d v
$$

The integral $I_{t}$ of Eq. (4) can be further transformed into an integral over the standard 2-square, $\{(\xi, \eta):-1 \leq \xi, \eta \leq 1\}$ using standard quadrilateral basis functions $Q_{i}(\xi, \eta)$, as shown in Fig. 3:

$$
\begin{aligned}
Q_{1}(\xi, \eta) & =\frac{1}{4}(\xi-1)(\eta-1), \\
Q_{2}(\xi, \eta) & =-\frac{1}{4}(\xi+1)(\eta-1), Q_{3}(\xi, \eta)=\frac{1}{4}(\xi+1)(\eta+1), \\
Q_{4}(\xi, \eta) & =-\frac{1}{4}(\xi-1)(\eta+1) .
\end{aligned}
$$

Assume that

$$
\begin{gathered}
u=\sum_{i=1}^{4} u_{i} Q_{i}=\frac{1}{4}(-1+3 \xi-\eta(1+\xi))=u(\xi, \eta) \\
v=\sum_{i=1}^{4} v_{i} Q_{i}=\frac{1}{4}(-1+3 \eta-\xi(1+\eta))=v(\xi, \eta)
\end{gathered}
$$

and

$$
J_{2}=\frac{\partial u}{\partial \xi} \frac{\partial v}{\partial \eta}-\frac{\partial u}{\partial \eta} \frac{\partial v}{\partial \xi}=\frac{1}{4}(2-\eta-\xi)
$$

Note that $J_{1}$ depends on the vertices of the given arbitrary triangular region, but $J_{2}$ is fixed.

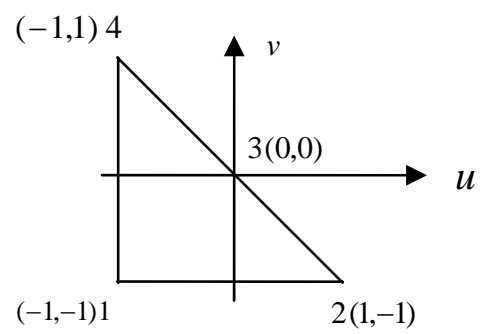

(a) Standard triangle, $S T$

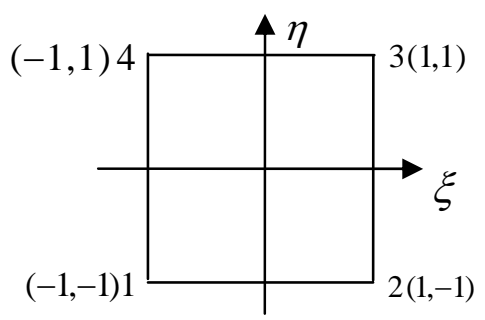

(b) Standard square, $S Q$

Fig. 3. Transformation of standard triangle $S T$ into 2-square $S Q$

Let $F(u, v)=f(u, v)\left|J_{1}\right|$ and using (5), Equ. (4) becomes

$$
\begin{gathered}
I_{t}=\int_{-1}^{1} \int_{-1}^{-u} F(u, v) d v d u=\int_{-1}^{1} \int_{-1}^{1} F(u(\xi, \eta), v(\xi, \eta))\left|J_{2}\right| d \xi d \eta \\
=\int_{-1}^{1} \int_{-1}^{1} F\left(\frac{1}{4}(-1+3 \xi-\eta(1+\xi)), \frac{1}{4}(-1+3 \eta-\xi(1+\eta))\right) \frac{1}{4}(2-\eta-\xi) d \xi d \eta \text { (6) }
\end{gathered}
$$

Now Eq. (6) represents an integral over the standard 2square region: $\{(\xi, \eta):-1 \leq \xi, \eta \leq 1\}$. Hence using Gauss Legendre quadrature rule for the integral $I_{t}$ of Eq. (6), we have

$I_{t}=\sum_{i=1}^{s} \sum_{j=1}^{s} \frac{1}{4}\left(2-\eta_{j}-\xi\right) w_{i} w_{j} F\left(\frac{1}{4}\left(-1+3 \xi-\eta_{j}(1+\xi)\right), \frac{1}{4}\left(-1+3 \eta_{j}-\xi\left(1+\eta_{j}\right)\right)\right)$

where $\left(\xi_{i}, \eta_{j}\right)$ are Gaussian points in the $\xi, \eta$ directions of order $s$ and $w_{i}, w_{j}$ are the corresponding weight coefficients. We can write Eq. (7) as:

$$
I_{t}=\sum_{k=1}^{N=s \times s} c_{k}^{\prime} F\left(x_{k}^{\prime}, y_{k}^{\prime}\right)
$$

where $c_{k}^{\prime}, x_{k}^{\prime}$ and $y_{k}^{\prime}$ can be written in the form:

$$
\begin{aligned}
& c_{k}^{\prime}=\frac{1}{4}\left(2-\eta_{j}-\xi_{i}\right) w_{i} w_{j} \\
& x_{k}^{\prime}=\frac{1}{4}\left(-1+3 \xi_{i}-\eta_{j}\left(1+\xi_{i}\right)\right) \\
& y_{k}^{\prime}=\frac{1}{4}\left(-1+3 \eta_{j}-\xi_{i}\left(1+\eta_{j}\right)\right) \\
& (k=1,2, \ldots \ldots \ldots, N),(i, j=1,2, \ldots \ldots \ldots \ldots, s)
\end{aligned}
$$


Table 1. Outputs of $c_{k}^{\prime}, x_{k}^{\prime}$ and $y_{k}^{\prime}$ of Eq.s (9) for $s=5$

\begin{tabular}{|c|c|c|c|}
\hline$k$ & $c_{k}^{\prime}$ & $x_{k}^{\prime}$ & $y_{k}^{\prime}$ \\
\hline 1 & 0.028067174431214 & -0.950889367642309 & 0.861470324235019 \\
\hline 2 & 0.046275406309135 & -0.758409434945362 & 0.686239721098985 \\
\hline 3 & 0.036857657161022 & -0.476544961484666 & 0.429634884453998 \\
\hline 4 & 0.015744196426143 & -0.194680488023970 & 0.173030047809011 \\
\hline 5 & 0.002633266629203 & -0.002200555327023 & -0.002200555327023 \\
\hline 6 & 0.067124593690865 & -0.942264702861852 & 0.502384453182495 \\
\hline 7 & 0.114542702111995 & -0.715982010624261 & 0.360956609587106 \\
\hline 8 & 0.099488780941957 & -0.384617327526421 & 0.153851982579262 \\
\hline 9 & 0.052864972328108 & -0.053252644428581 & -0.053252644428581 \\
\hline 10 & 0.015744196426143 & 0.173030047809011 & -0.194680488023970 \\
\hline 11 & 0.097927415226499 & -0.929634884453998 & -0.023455038515334 \\
\hline 12 & 0.172797751608793 & -0.653851982579262 & -0.115382672473579 \\
\hline 13 & 0.161817283950617 & -0.250000000000000 & -0.250000000000000 \\
\hline 14 & 0.099488780941957 & 0.153851982579262 & -0.384617327526421 \\
\hline 15 & 0.036857657161022 & 0.429634884453998 & -0.476544961484666 \\
\hline 16 & 0.097655803573857 & -0.917005066046144 & -0.549294530213163 \\
\hline 17 & 0.176220431895882 & -0.591721954534264 & -0.591721954534264 \\
\hline 18 & 0.172797751608793 & -0.115382672473579 & -0.653851982579262 \\
\hline 19 & 0.114542702111995 & 0.360956609587106 & -0.715982010624261 \\
\hline 20 & 0.046275406309135 & 0.686239721098985 & -0.758409434945362 \\
\hline 21 & 0.053501082233226 & -0.908380401265687 & -0.908380401265687 \\
\hline 22 & 0.097655803573857 & -0.549294530213163 & -0.917005066046144 \\
\hline 23 & 0.097927415226499 & -0.023455038515334 & -0.929634884453998 \\
\hline 24 & 0.067124593690865 & 0.502384453182495 & -0.942264702861852 \\
\hline 25 & 0.028067174431214 & 0.861470324235019 & -0.950889367642309 \\
\hline
\end{tabular}

The weighting coefficients $c_{k}^{\prime}$ and sampling points $\left(x_{k}^{\prime}, y_{k}^{\prime}\right)$ of various order can be now easily computed from Eq.(9). Using Mathematica program, the outputs of $c_{k}^{\prime}, x_{k}^{\prime}$ and $y_{k}^{\prime}$ for $s=5$ are given in Table 1 .

Now we decompose $S T=\{(u, v):-1 \leq v \leq 1,-1 \leq u \leq-v\}$ in $(u, v)$-space of Eq. (4) into $4(n \times n)=4 n^{2}$ right isosceles triangles, $T_{i}$ with side lengths $1 / n^{10-13}$. Then Eq. (4) reduces to

$$
I_{t}=\sum_{i=1}^{4(n \times n)} \iint_{T_{i}} F(u, v) d u d v
$$

Since each $T_{i}$ is to be transformed again into a standard triangle and using composite integration rule $e^{10-13}$ we can obtain the following:

$$
I_{t}=\frac{1}{4 n^{2}} \sum_{k=1}^{N=s \times s} c_{k}^{\prime} H\left(x_{k}^{\prime}, y_{k}^{\prime}\right)
$$

where

$$
\begin{aligned}
& H\left(x_{k}^{\prime}, y_{k}^{\prime}\right)=\sum_{i=0}^{2 n-1} \sum_{j=0}^{2 n-1-i} F\left(\frac{x_{k}^{\prime}+2(i-n)+1}{2 n}, \frac{y_{k}^{\prime}+2(j-n)+1}{2 n}\right) \\
& +\sum_{i=0}^{2 n-22 n-2-i} \sum_{j=0} F\left(\frac{-x_{k}^{\prime}+2(i-n)+1}{2 n}, \frac{-y_{k}^{\prime}+2(j-n)+1}{2 n}\right)
\end{aligned}
$$

and

$$
\begin{aligned}
& c_{k}^{\prime}=\frac{1}{4}\left(2-\xi_{p}-\eta_{q}\right) w_{p} w_{q} \\
& x_{k}^{\prime}=\frac{1}{4}\left(-1+3 \xi_{p}-\eta_{q}\left(1+\xi_{p}\right)\right) \\
& y_{k}^{\prime}=\frac{1}{4}\left(-1+3 \eta_{q}-\xi_{p}\left(1+\eta_{q}\right)\right) \\
&(k=1,2, \ldots \ldots \ldots \ldots, N),(i, j=1,2, \ldots \ldots \ldots \ldots
\end{aligned}
$$

\section{Numerical Examples}

In this section, all examples have taken from the recent paper ${ }^{14}$ which are shown in Tables 2-4. 


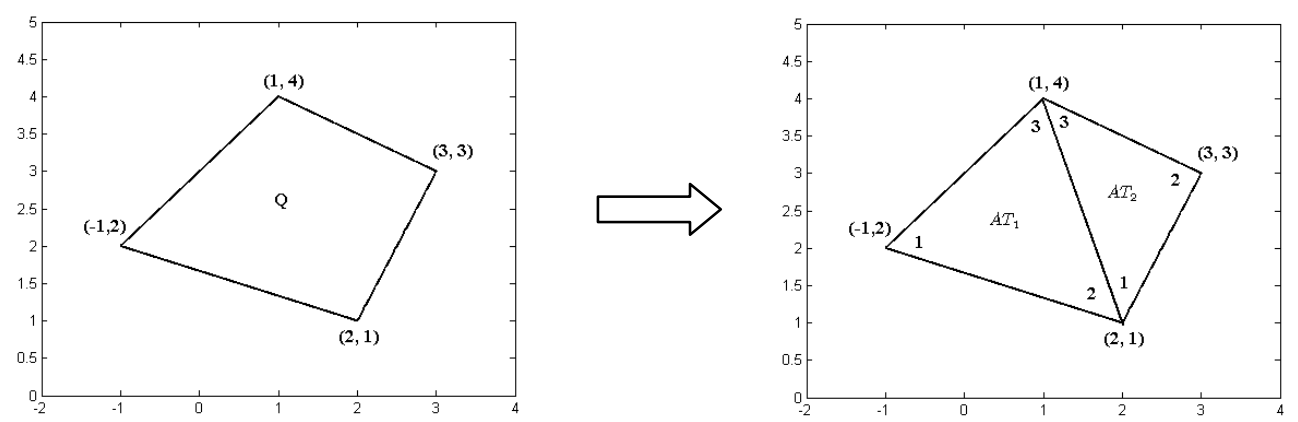

Fig. 4. Quadrilateral region $Q$
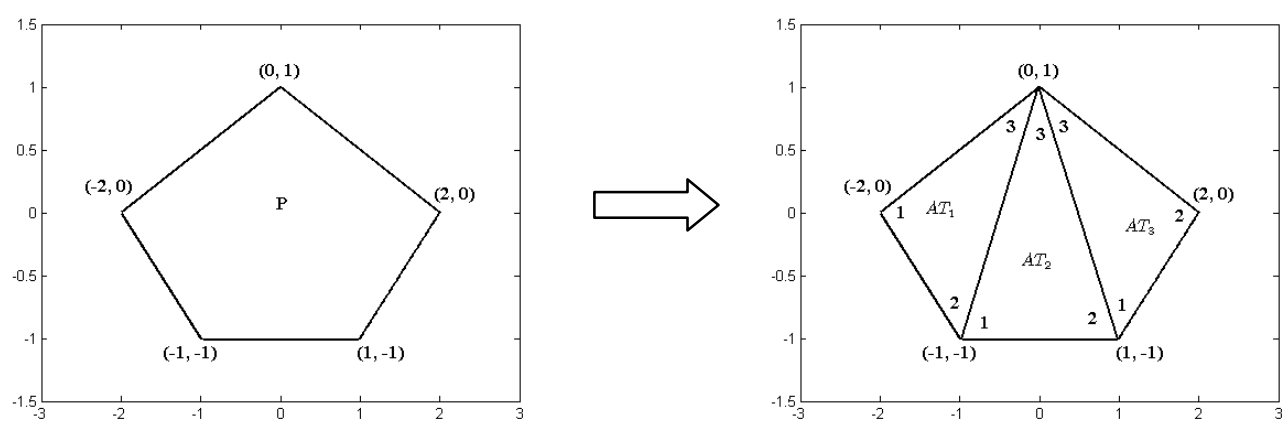

Fig. 5. Pentagon $P$

Table 2. Generalized composite numerical integration over triangular, rectangular and parallelogram regions

\begin{tabular}{|c|c|c|c|c|}
\hline Integral with exact value & $S$ & $\begin{array}{l}\text { Computed value using } \\
\text { present method for } \\
\left(4 \times 5^{2}\right) \text { sub triangles }\end{array}$ & $\begin{array}{l}\text { Computed value using } \\
\text { present method for } \\
\left(4 \times 10^{2}\right) \text { sub triangles }\end{array}$ & $\begin{array}{l}\text { Computed value using } \\
\text { the method of Sarada } \\
\text { and Nagaraja }{ }^{14}\end{array}$ \\
\hline \multirow{3}{*}{$\begin{array}{l}\int_{0}^{1} \int_{x}^{2-x} \sqrt{x+y} d y d x \\
=1.13137084989848\end{array}$} & $s=3$ & 1.131372465703519 & 1.131371135535597 & \\
\hline & $s=5$ & 1.131371010152331 & 1.131370878227623 & 1.13137111584312 \\
\hline & $s=10$ & 1.131370849405025 & 1.131370849898650 & 1.13137084930298 \\
\hline \multirow{3}{*}{$\begin{array}{l}\int_{1}^{3} \int_{1-x}^{2 x-2}\left[(x+y)^{1 / 2}(1+x+y)^{2}\right] d y d x \\
=211.418375377476\end{array}$} & $s=3$ & 211.418375413220700 & 211.418375378138600 & \\
\hline & $s=5$ & 211.418375377477900 & 211.418375377475800 & 211.418375371115 \\
\hline & $s=10$ & 211.418375377475800 & 211.418375377475700 & 211.418375377482 \\
\hline \multirow{2}{*}{$\int_{3}^{5} \int_{1}^{4} \sqrt{x+y} d x d y$} & $s=3$ & 15.247081338038640 & 15.247081338037520 & \\
\hline & $s=5$ & 15.247081338037510 & 15.247081338037510 & 15.2470813595701 \\
\hline$=15.2470813380375$ & $s=10$ & 15.247081338037510 & 15.247081338037510 & 15.2470813380375 \\
\hline \multirow{3}{*}{$\begin{array}{l}\int_{1}^{2} \int_{-1}^{3-x} \sqrt{x^{2}+y^{2}} d y d x \\
=4.16872178857775\end{array}$} & $s=3$ & 4.168721788528673 & 4.168721788576948 & \\
\hline & $s=5$ & 4.168721788577755 & 4.168721788577756 & 4.16896959209620 \\
\hline & $S=10$ & 4.168721788577755 & 4.168721788577755 & 4.16872193734188 \\
\hline \multirow{2}{*}{$\int_{0}^{1} \int_{1-x}^{2+x}(x+y)^{1 / 2}(1+x+y)^{2} d y d x$} & $s=3$ & 30.631252208539310 & 30.631252208190520 & \\
\hline & $s=5$ & 30.631252208184680 & 30.631252208184680 & 30.6312523036460 \\
\hline$=30.6312522081847$ & $s=10$ & 30.631252208184680 & 30.631252208184680 & 30.6312522081853 \\
\hline \multirow{3}{*}{$\begin{array}{l}\int_{-1}^{0} \int_{-y-2}^{y+2} \frac{x^{4}+y^{3}}{1+x^{2}} d x d y \\
=1.00601339577510\end{array}$} & $s=3$ & 1.006013394580186 & 1.006013395754989 & \\
\hline & $s=5$ & 1.006013395775123 & 1.006013395775098 & 0.99528697888712 \\
\hline & $s=10$ & 1.006013395775098 & 1.006013395775098 & 1.00637151173877 \\
\hline
\end{tabular}


Table 3. Generalized composite numerical integration over arbitrary quadrilateral region $Q$ (Fig.4)

\begin{tabular}{|c|c|c|c|c|}
\hline Integral with exact value & $S$ & $\begin{array}{l}\text { Computed value using } \\
\text { present method for } \\
\left(4 \times 5^{2}\right) \text { sub triangles }\end{array}$ & $\begin{array}{l}\text { Computed } r \text { value } \\
\text { using present method } \\
\text { for }\left(4 \times 10^{2}\right) \text { sub } \\
\text { triangles }\end{array}$ & $\begin{array}{l}\text { Computed value using } \\
\text { the method of Sarada } \\
\text { and Nagaraja }{ }^{14}\end{array}$ \\
\hline $\iint\left[(x+y)^{1 / 2}(1+x+y)^{2}\right] d y d x$ & $s=3$ & 298.234339210737800 & 298.234339210045600 & \\
\hline & $s=5$ & 298.234339210034300 & 298.234339210034300 & 298.234338347174 \\
\hline$=298.234339210034$ & $s=10$ & 298.234339210034300 & 298.234339210034300 & 298.234339210033 \\
\hline $\iint_{-}$ & $s=3$ & 3.549613019979926 & 3.549613026675375 & \\
\hline $\int_{Q} \sqrt{x+y}$ & $s=5$ & 3.549613026789603 & 3.549613026789717 & 3.54960971225221 \\
\hline$=3.54961302678971$ & $s=10$ & 3.549613026789717 & 3.549613026789717 & 3.54961302681661 \\
\hline
\end{tabular}

Table 4. Generalized composite numerical integration over any arbitrary pentagon $P$ (Fig. 5)

\begin{tabular}{|c|c|c|c|c|}
\hline Integral with exact value & $S$ & $\begin{array}{l}\text { Computed value using } \\
\text { present method for } \\
\left(4 \times 5^{2}\right) \text { sub triangles }\end{array}$ & $\begin{array}{l}\text { Computed value using } \\
\text { present method for } \\
\left(4 \times 10^{2}\right) \text { sub triangles }\end{array}$ & $\begin{array}{l}\text { Computed value using the } \\
\text { method of Sarada and } \\
\text { Nagaraja }^{14}\end{array}$ \\
\hline \multirow{2}{*}{$\iint_{P}(1-x) \sin (10 x y) d x d y$} & $s=3$ & -0.013100041165331 & \multicolumn{2}{|l|}{-0.013103628437347} \\
\hline & $s=5$ & - 0.013103721381516 & -0.013103719671051 & - 0.451592814601184 \\
\hline$=-0.013103719669957$ & $s=10$ & -0.013103719669958 & -0.013103719669957 & - 0.018237768238161 \\
\hline $\iint x^{4}+y^{3}$ & $s=3$ & 1.924030542963068 & 1.924030542637816 & \\
\hline $\iint_{P} \frac{x+y}{1+x^{2}} d x d y$ & $s=5$ & 1.924030542632652 & 1.924030542632650 & 1.90714805151570 \\
\hline$=1.92403054263265$ & $s=10$ & 1.924030542632650 & 1.924030542632651 & 1.92457441414507 \\
\hline
\end{tabular}

\section{Conclusions}

In this paper, we have discussed the formulation of double integrals over an arbitrary polygon. At first we have decomposed any polygon with $m$-sides into $m-2$ triangles. Each triangular region is transformed into a standard triangle $\{(u, v):-1 \leq u \leq 1,-1 \leq v \leq-u\}$ by using triangular basis functions. Each of the standard triangles is further decomposed into $4 \times n^{2}$ triangles. Then we map each of the standard triangle into the 2-square using standard quadrilateral basis functions. For each triangle we generate $s^{2}$ new Gauss points using the lower order conventional Gauss quadrature of order $s$, and thus the composite numerical integration over the standard triangular finite elements are applied. We observe that computed results of the given examples converge to the exact solutions correct upto fifteen decimal places. The technique of this paper, using lower order Gauss Legendre quadrature rule, can give high accuracy results compare to the existing formulations in the literatures.

\section{References}

1. Hammer, P. C., O. J. Marlowe and A. H. Stroud, 1956. Numerical integration over simplexes and cones. Math. Tables and other aids to computation, 10, 130-136.

2. Hammer, P. C. and A. H. Stroud, 1956. Numerical integration over simplexes. Math. Tables and other aids to computation, 10, 137-139.

3. Hammer, P. C. and A. H. Stroud, 1958. Numerical evaluation of multiple integrals. Math. Tables and other aids to computation, 12, 272-280.
4. Stroud, A. H., 1974. Numerical Quadrature and Solution of Ordinary Differential Equations, Springer-Verlag, New York Berlin Heidelberg.

5. Zienkiewicz, O. C., 1977. The Finite Element Method, 3rd Edn., Mc Graw Hill Inc.

6. Cowper, G. R., 1973. Gaussian quadrature formulas for triangles. Inter. Jn. Numer. Methods Engrs, 7 , 405-408.

7. Lethor, F. G., 1976. Computation of double integrals over a triangle. J. Computational and Applied Mathematics, 2, 219-224

8. Hillion, P., 1977. Numerical integration on a triangle, Inter. Jn. Numer. Methods Engrs. 11, 797-815.

9. Lauresn, M. E. and M. Gellert, 1978. Some criteria for numerically integrated matrices and quadrature formulas for triangles, Inter. Jn. Numer. Methods Engrs. 12, 67-76.

10. Rathod, H.T., K.V. Nagaraja and B. Venkatesudu, 2007. Symmetric Gauss Legendre quadrature formulas for composite numerical integration over a triangular surface. Appl. Math. Comput., 188, 865-876.

11. Rathod, H.T., K.V. Nagaraja and B. Venkatesudu, 2007. On the application of two symmetric Gauss Legendre quadrature rules for composite numerical integration over a triangular surface. Appl. Math. Comput., 190, 21-39.

12. Islam, M. S. and M. A. Hossain, 2008. Numerical Integration over an Arbitrary Triangular Region. International e-Journal of Numerical Analysis and Related Topics (IeJNART), 2, 25-40.

13. Islam, M. S. and M. A. Hossain, 2009. Numerical Integrations over an Arbitrary Quadrilateral Region. Appl. Math. Comput., 210, $515-524$.

14. Sarada, J. and K.V. Nagaraja, 2011. Generalized Gaussian quadrature rules over two-dimensional regions with linear sides. Appl. Math. Comput., 217, 5612-5621. 

Dhaka Univ. J. Sci. 62(1): 25-29, 2014 (January)

*Author for Correspondence. e-mail: mdshafiqul_mat@du.ac.bd 
Dhaka Univ. J. Sci. 62(1): 25-29, 2014 (January) 
